\title{
The Microbiome in Critical Illness: Firm Conclusions or Bact to Square One?
}

\author{
John D. Lyons ${ }^{1} \cdot$ Mandy L. Ford ${ }^{2}$ Craig M. Coopersmith ${ }^{1}$
}

Published online: 29 February 2016

(C) Springer Science+Business Media New York 2016

The microbiome is a vast collection of human-associated bacterial communities residing within the gastrointestinal lumen, mouth, skin, and all surfaces exposed to the external environment [1]. Hundred trillion bacteria are estimated within the intestinal tract and on the surfaces of the human body, meaning the microbiome has 10 times more cells than are of host origin [2]. Further, for every gene in the human genome, it is estimated that there are 100 bacterial genes. Philosophically, this might result in reflection as to what defines "self," since, in actuality, human host cells and DNA make up the minority population even within our own bodies. Practically, modern medicine has only recently begun to consider how events within this single-celled world may scale up to impact the multicellular host. These investigations have revealed an ever-unfolding contribution of the microbiome to biological processes as diverse as postoperative healing, uveitis, and the response to cancer therapy [3-6]. Intriguingly, increasing knowledge of microbial populations in disease has emphasized that the complexities of the microbiome and its interactions with our bodies may have far-reaching consequences.

The microbiome has increasingly become an important focus of research in critical illness, both as a target of potential therapeutics as well as to obtain a mechanistic

Craig M. Coopersmith

cmcoop3@emory.edu

1 Emory Critical Care Center and Department of Surgery, Emory Healthcare and Emory University School of Medicine, 101 Woodruff Circle, Suite WMB 5105, Atlanta, GA 30322, USA

2 Emory Transplant Center and Department of Surgery, Emory Healthcare and Emory University School of Medicine, 101 Woodruff Circle, Suite WMB 5105, Atlanta, GA 30322, USA understanding of how our internal bacteria help shape our response to acute illness. Augmenting "beneficial" bacteria (via probiotics, synbiotics, or prebiotics) or decreasing "harmful" bacteria (via selective decontamination of the digestive system) could potentially improve outcomes in critically ill patients. Both of these approaches have some potential clinical merit, although substantive concerns regarding either efficacy or safety have prevented them from being widely utilized in clinical practice. Furthermore, it is difficult to know how best to target the microbiome therapeutically when the understanding of this complex ecosystem is still in a relatively nascent state.

In this month's issue of Digestive Diseases and Sciences, Ojima et al. [7] begin to address this knowledge gap with a pilot study on the effect of ICU admission for acute critical illness on the bacterial composition within the microbiome. The authors prospectively monitored 12 mechanically ventilated adult patients at a large, academic hospital in Japan, taking serial stool samples from patients and comparing them to those collected from healthy controls. Utilizing 16S rRNA metagenomic sequencing, they determined the relative proportions of five major enteric bacterial phyla (Bacteroidetes, Firmicutes, Proteobacteria, Actinobacteria, and Fusobacteria). The authors observed dynamic changes in the composition of the microbiome in all 12 patients, most experiencing a significant change in just the first 3-5 days after admission. Moreover, the Bacteroidetes/Firmicutes ratio was substantially skewed in the 5/6 patients who died (greater than 10:1 in four patients and less than 1:10 in one patient). In contrast, the ratio was not altered in any of the survivors, suggesting a possible association of this bacterial imbalance with ICU mortality.

This study builds upon previous data from the same group examining the microbiome via bacterial culturebased methodologies in patients with the systemic 
inflammatory response syndrome [8, 9]. Nonetheless, it expands on prior studies in new and important ways. First, rather than looking at a single snapshot in time, the study examined multiple time points for each patient, demonstrating how rapidly the microbiome changes in response to critical illness and therapies initiated in the ICU. The absolute components of the microbiome, altered as early as 1 day after ICU admission, changed considerably on a near-daily basis for the first week of ICU stay. In contrast, the components of the microbiome as well as the ratio of Bacteroidetes/Firmicutes were relatively constant over the same time period in concurrently studied control population, consistent with the general stability of the microbiome in healthy individuals [10]. Still, previous studies of the microbiome in the ICU were limited by conventional culture methods, which are unable to reliably identify anaerobes, which lose their symbiotic relationship once outside the body. The metagenomic approach used by Ojima et al. enabled a more comprehensive analysis of obligate anaerobes and other microbes than was previously possible, a great strength of the study.

Like most studies, however, this report raises more questions than it answers. As a pilot study on a very small patient population, there is no way to know whether the results are generalizable. Further, while all patients were receiving antibiotics, the antibiotic regimen varied between patients, which adds a major uncontrolled variable since each antibiotic has unique antibacterial specificities, which adds doubt as to which intervention was more important: critical illness or antibiotic administration, a question that could be addressed by studying the microbiome of a noncritical population treated with similar antibiotics. Further, the contribution of other variables such as primary diagnosis in the ICU, gender, age, feeding status, comorbidities, pre-morbid state, and severity of illness among others cannot be determined in this study.

By virtue of its observational nature, data acquired from this study cannot be used to determine how_or if — changes in the microbiome impact outcomes in the ICU. Yet, recent literature addressing the influence of the microbiome on cancer therapy may provide tantalizing hints about potential contributions. The efficacy of the checkpoint inhibitors programmed-death ligand-1 (PDL1) and anticytotoxic lymphocyte antigen-4 (CTLA-4) on treatment efficacy in murine models of cancer is dependent on the presence or absence of specific bacterial strains within the microbiome $[5,6]$. Components of the microbiome activate the immune system, rendering it more effective at attacking tumor cells. If the microbiome is capable of influencing the response of immune cells to specific stimuli and antigens in cancer, it is also theoretically possible that changes in the makeup of the microbiome may bolster or hamper the immune system of critically ill patients. Understanding the microbiome as a potential controller of the host response-instead of as a bystander-has tremendous implications for the care of critical illness; an understanding of how critical illness affects specific bacteria over a defined time course is an important first step in the quest to harness the power of the trillions of organisms that live inside us.

Acknowledgments This work was supported by funding from the National Institutes of Health (GM072808, GM095442, GM104323, GM109779, GM113228).

\section{References}

1. Defazio J, Fleming ID, Shakhsheer B, Zaborina O, Alverdy JC. The opposing forces of the intestinal microbiome and the emerging pathobiome. Surg Clin North Am. 2014;94:1151-1161.

2. Ley RE, Peterson DA, Gordon JI. Ecological and evolutionary forces shaping microbial diversity in the human intestine. Cell. 2006; 124:837-848.

3. Shogan BD, Belogortseva N, Luong PM, et al. Collagen degradation and MMP9 activation by Enterococcus faecalis contribute to intestinal anastomotic leak. Sci Transl Med. 2015;7:286ra68.

4. Horai R, Zarate-Blades CR, Dillenburg-Pilla P, et al. Microbiotadependent activation of an autoreactive $\mathrm{T}$ cell receptor provokes autoimmunity in an immunologically privileged site. Immunity. 2015;43:343-353.

5. Sivan A, Corrales L, Hubert N, et al. Commensal Bifidobacterium promotes antitumor immunity and facilitates anti-PD-L1 efficacy. Science. 2015;350:1084-1089.

6. Vetizou M, Pitt JM, Daillere R, et al. Anticancer immunotherapy by CTLA-4 blockade relies on the gut microbiota. Science. 2015;350:1079-1084.

7. Ojima M, Motooka D, Shimizu K, et al. Metagenomic analysis reveals dynamic changes of whole gut microbiota in the acute phase of intensive care unit patients. Dig Dis Sci. (Epub ahead of print). doi:10.1007/s10620-015-4011-3.

8. Shimizu K, Ogura H, Goto M, et al. Altered gut flora and environment in patients with severe SIRS. J Trauma. 2006;60: 126-133.

9. Shimizu K, Ogura H, Hamasaki T, et al. Altered gut flora are associated with septic complications and death in critically ill patients with systemic inflammatory response syndrome. Dig Dis Sci. 2011;56:1171-1177.

10. Alverdy J, Gilbert J, Defazio JR, et al. Proceedings of the 2013 A.S.P.E.N. Research workshop: the interface between nutrition and the gut microbiome: implications and applications for human health [corrected]. JPEN. 2014;38:167-178. 